\title{
Reconstrucción de la agricultura prehispánica en El Salvador previo a la erupción volcánica, a través del análisis de suelos
}

DOI: http://dx.doi.org/10.5377/koot.v0i9.5903

URI: http://hdl.handle.net/11298/433

\author{
Nobuyuki Ito, Takeshi Watanabe y Makoto Kimura \\ Arqueólogo, Catedrático e investigador \\ Universidad Nagoya, Japón \\ nob9mex@yahoo.co.jp
}

\section{Resumen}

En El Salvador se han realizado hallazgos arqueológicos substanciales relacionados con las prácticas de agricultura prehispánica en diversos sitios arqueológicos. En Joya de Cerén se encontraron muy bien conservadas evidencias de surcos de milpas correspondientes al periodo Clásico Tardío, así como cultivos de maíz, frijoles, chiles, maguey, cacao, yuca, y otros. En el Antiguo Cuscatlán, abajo de una capa de tefra de Cuzcatán (900-650 a.C.) hallaron varios surcos y zanjas, en las cuales se encontró un macrofósil de hoja de maíz con un fechamiento de 820 a.C. Por otra parte, en los sitios arqueológicos, como Cerrón Grande, Bulevar Monseñor Oscar Arnulfo Romero, Chalchuapa, se reportaron surcos abajo la capa de ceniza volcánica de Ilopango (T.B.J./siglo IV-VI d.c.), aunque no se informó que tipo de plantas se cultivaron ahí.

El humus o sostenimiento húmico del suelo se constituye por una capa de tierra fértil de color obscuro, la cual se forma a través de los residuos y descomposición de plantas originarias que se desarrollan y mueren en la superficie. Por esta razón, el valor de $\delta 13 \mathrm{C}(\%)$ del suelo es variable, dependiendo del tipo de plantas que crecieron en el pasado.

Se ha realizado el análisis de valor de $\delta 13 \mathrm{C}$ a través del analizador de masa de isótopos estables para identificar los cultivos de la época anterior a la erupción volcánica del Ilopango, ya que no se ha encontrado ningún material de flora o de cultivo. Por análisis de valor de $\delta 13 \mathrm{C}$, se puede decir que se cultivaba plantas del tipo C4 en la milpa de Bulevar Monseñor Oscar Arnulfo Romero.

Palabras clave: Agricultura primitiva, Agricultura - cultivo - historia - El Salvador, Sociedades primitivas, Indígenas de El Salvador, Etnobotánica, Culturas indígenas. 


\begin{abstract}
In El Salvador, substantial archaeological findings have been made related to pre-Hispanic agriculture practices in various archaeological sites. In Joya de Cerén, there were found very well-conserved evidences of furrows of maize fields corresponding to the Late Classic Period, as well as crops of corn, beans, maguey, cocoa, cassava and others. In Antiguo Cuscatlan, under a layer of tephra from Cuzcatan (900-650 BC), several grooves and ditches that included a macrofossil with a corn leaf dating to $820 \mathrm{BC}$ were found. On the other hand, archaeological sites, such as Cerrón Grande, Monseñor Oscar Arnulfo Romero Boulevard, Chalchuapa, reported grooves under the volcanic ash layer of Ilopango (TBJ / century IV-VI AC) although it was not reported the type of plants that there were grown there.
\end{abstract}

The humus or humic support of the soil is constituted by a layer of fertile earth of dark color, which is formed through the remains and decomposition of native plants that develop and die on the surface. For this reason, the value of $\delta 13 \mathrm{C}(\mathrm{o} / \mathrm{oo})$ of the soil is variable, depending on the type of plants that grew in the past.

The value analysis has been carried out $\delta 13 \mathrm{C}$ through the mass analyzer of stable isotopes to identify the crops of the period before the volcanic eruption of Ilopango, since no material of vegetation or crop has been found. By value analysis $\delta 13 \mathrm{C}$, it can be stated that $\mathrm{C} 4$ type plants were cultivated in the maize fields of Bulevar Monseñor Oscar Arnulfo Romero.

Keywords: Primitive agriculture, Agriculture - crops - history - El Salvador, Primitive societies, Indigenous people of El Salvador Ethnobotany, Indigenous cultures.

\title{
Introducción
}

En la República de El Salvador se han realizado hallazgos arqueológicos substanciales relacionados con las prácticas de agricultura prehispánica en diversos sitios arqueológicos (Fig. 1). Ejemplo de lo anterior es el sitio arqueológico Joya de Cerén, en el cual se encontraron muy bien conservados surcos de milpas correspondientes al período Clásico Tardío, así como cultivos de maíz, frijoles, chiles, maguey, cacao, yuca y otros (Sheets, 2002).

Por otra parte, en el sitio de Cerrón Grande debajo de la capa de ceniza volcánica del Ilopango, conocida como Tierra Blanca Joven (T.B.J.), se reportaron surcos pertenecientes a un período más temprano que el de Joya del Cerén, aunque no se mencionó que tipo de plantas se cultivaron ahí. De igual forma, en el área de Casa Blanca, Chalchuapa se localizaron surcos en la orilla Este del "Gran Basamento".

En Boulevard Monseñor Romero, San Salvador, desde hace unos años dio inicio la construcción de nuevas carreteras. Durante el proceso de la construcción, 
se cortaron algunas lomas y ciertos terrenos. En dichos cortes se evidenciaron surcos debajo de la T.B.J.

El humus se constituye por una capa de tierra fértil de color obscuro, la cual se forma a través de los residuos y descomposición de plantas originarias que se desarrollan y mueren en la superficie. Por esta razón, el valor de $\delta 13 \mathrm{C} \mathrm{( \% o)} \mathrm{del}$ suelo es variable, dependiendo del tipo de plantas que crecieron en el pasado. El suelo formado por plantas $\mathrm{C} 4$ muestra más alto valor de $\delta 13 \mathrm{C}$ que el de plantas $\mathrm{C} 3$. Cuando las plantas $\mathrm{C} 4$ crecen continuamente, el valor de $\delta 13 \mathrm{C}$ del suelo se incrementa por el tiempo. Si el suelo presenta un alto valor de $\delta 13 \mathrm{C}$, indica que las plantas $\mathrm{C} 4$ pudiesen haber crecido por cierto tiempo en el campo o la milpa.

De esta forma fue que en los surcos abajo de la ceniza volcánica de Ilopango dio inicio la investigación de la agricultura prehispánica a través del reconocimiento del suelo, no obstante que no se ha encontrado a la fecha, ningún resto vegetal del cultivo, como semillas, hojas o tallos.

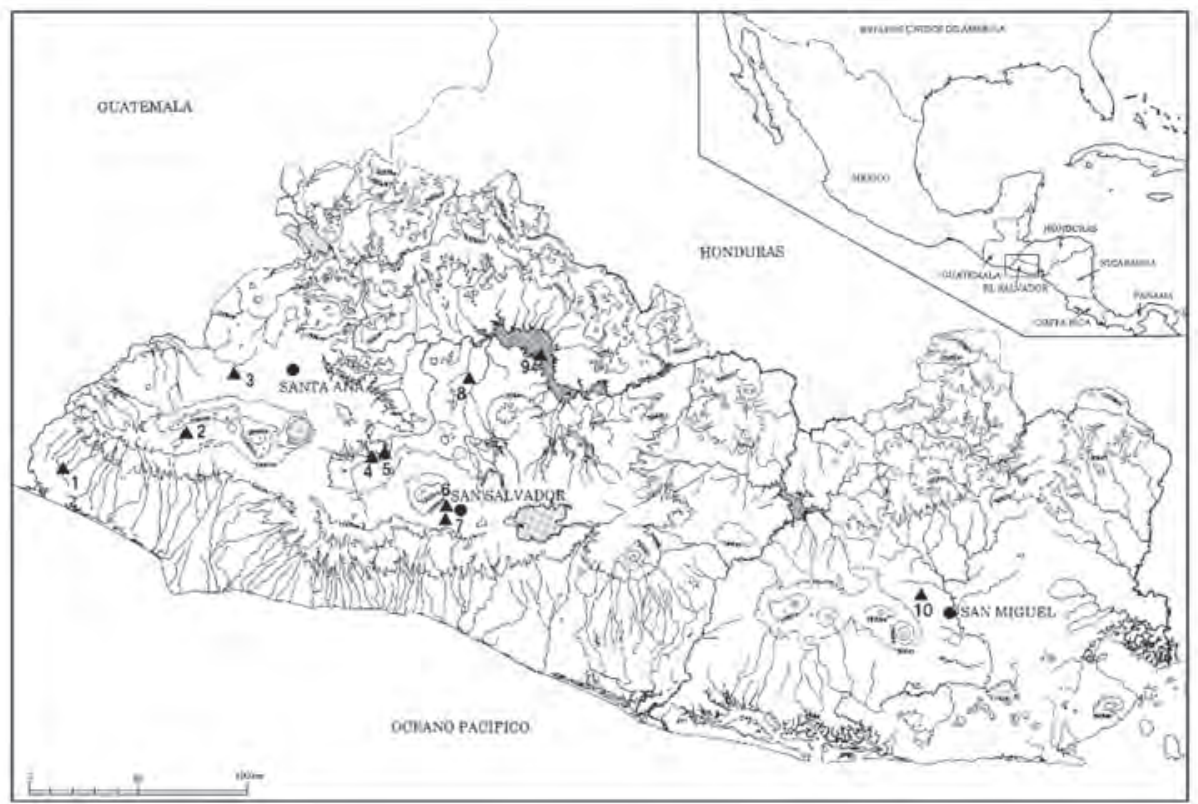

Figura 1. Sitios arqueológicos principales y agrícolas de El Salvador: 1. Cara Sucia, 2. Santa Leticia, 3. Chalchuapa, 4. San Andrés, 5. Joya de Cerén, 6. Boulevard Monseñor Romero, 7. Antiguo Cuscatlán, 8. Cihuatan, 9. Cerrón Grande, 10. Quelepa

\section{Polen de Casa Blanca, Chalchuapa}

En el área de Casa Blanca, Chalchuapa, se tomaron algunas muestras de tierra para analizar la flora o los cultivos existentes antes de la erupción del Volcán Ilopango (Fig. 2). Se realizó la flotación de tierra de la capa correspondiente a los 
surcos sin localizar semillas de ninguna especie de plantas. También se realizó el análisis de polen y en términos generales se puede decir que la cantidad de palinomorfos recuperada fue muy baja, por lo que aún no es posible discernir en ningún tipo de interpretación, aunque se encontraron algunas partículas de planta. El estrato 5, correspondiente a la capa donde se encontraron los surcos prehispánicos, se localizó un grano que podría ser de Zea mays (Xelhuantzi, López 2010); sin embargo, no se puede decir con ninguna certeza que allí se cultivó maíz.
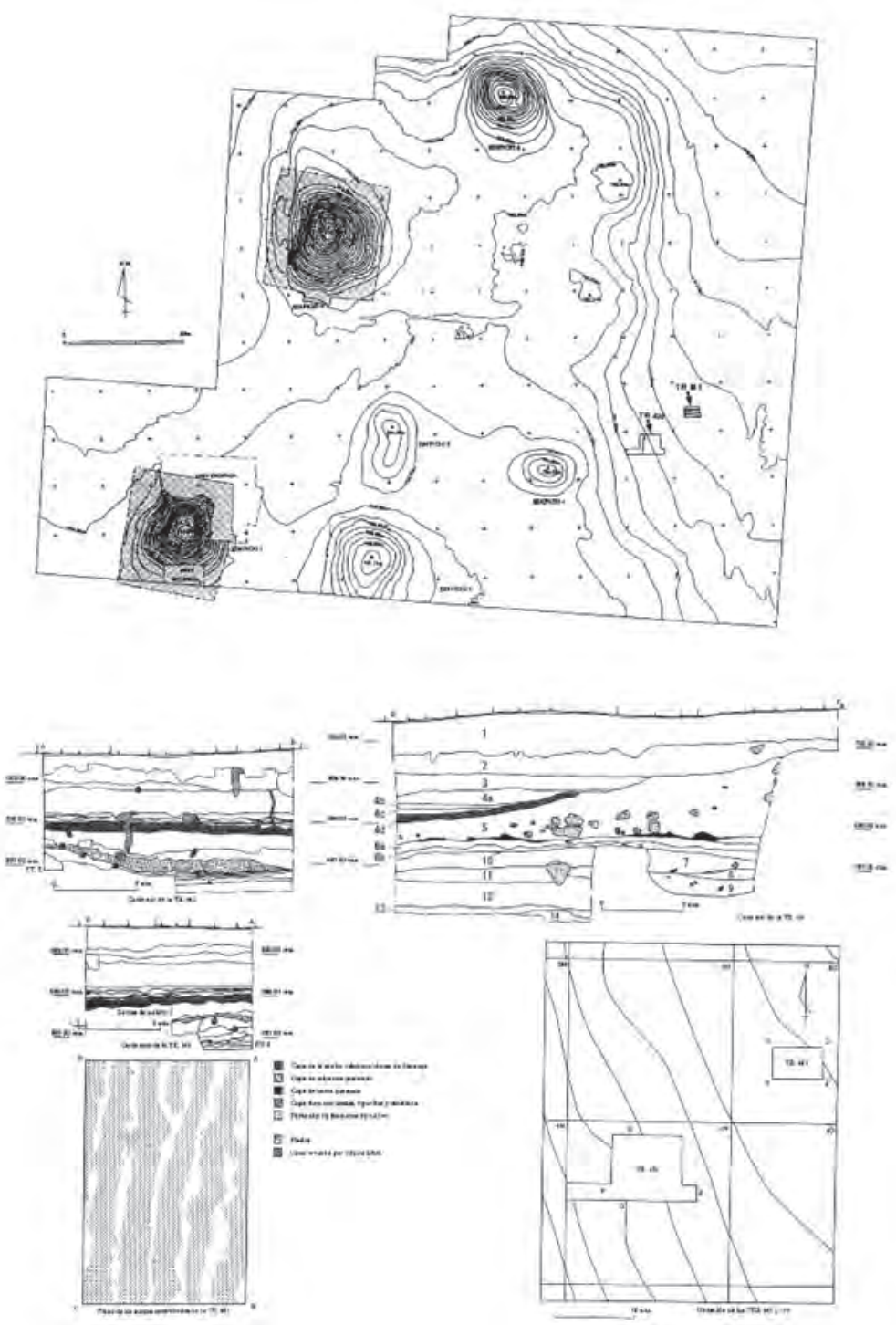

Figura 2. Surcos de Casa Blanca, Chalchuapa 


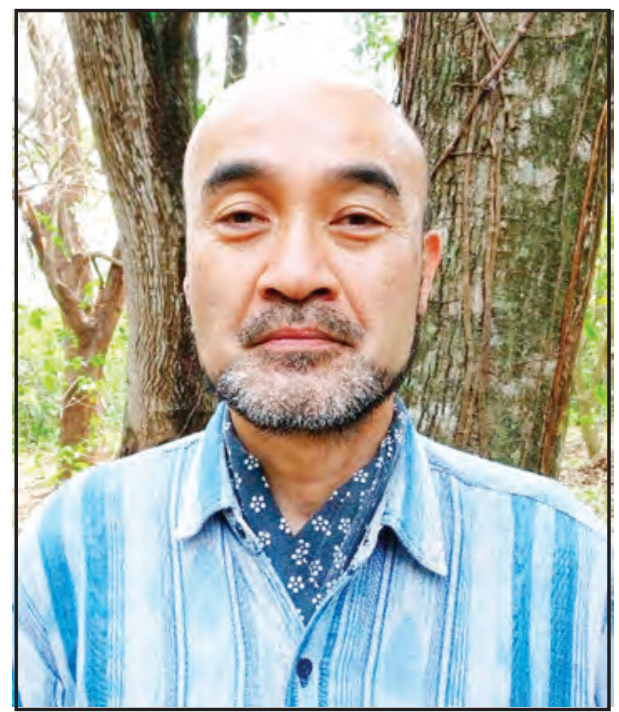

Doctor y Arqueólogo Nobuyuki Ito

\section{Surcos prehispánicos}

En toda Mesoamérica se reportan surcos de milpa prehispánicos. Ejemplos de ello se muestran en el Altiplano Central de México, principalmente en el sitio arqueológico de Tetimpa, en Puebla, en el que reporta el hallazgo de agricultura prehispánica abajo de la capa volcánica (Plunket y Uruñuela, 1998). Sitios comparativamente similares se localizan en El Salvador, como lo son Cerrón Grande, Chalchuapa, Antiguo Cuscatlán y Boulevard Monseñor Romero.

En el sitio Cerrón Grande, abajo de la capa de ceniza volcánica de T.B.J, se localizaron surcos con un ancho de $1.00 \mathrm{~m}$ aproximadamente sin reportar el tipo de cultivo prehispánico (Earnest, 1976). En el Antiguo Cuscatlán, hallaron varios surcos en diferentes niveles estratigráficos. Abajo de la ceniza volcánica de T.B.J., las cunetas tienen un ancho de $0.61-1.47 \mathrm{~m}$ con un promedio de 0.996 $\mathrm{m}$ y no se reportó ningún hallazgo de cultivos. Más abajo se encontró una capa de tefra de Cuzcatán, la cual podría provenir del cráter de Plan de La Laguna. Por el estudio de los materiales arqueológicos se fechó como de la fase Colos de Chalchuapa, entre 900 y 650 AC. Abajo de esta capa de tefra, se encontraron unos surcos con una anchura de $0.676 \mathrm{~m}$ entre los surcos. El suelo de surcos es rojizo y arcilloso muy meteorizado. También en la zanja se encontró un macrofósil de hoja de maíz, el cual tiene un fechamiento de 820 a.C. $(1 \delta=895$ 800 AC) (Amalori, 1999).

En los sitios arqueológicos de Chalchuapa y Boulevard Monseñor Romero se han encontrado unos surcos prehispánicos abajo de T.B.J. sin ninguna evidencia del cultivo. 


\section{Análisis de suelo}

\section{Las plantas de C3, C4 y Cam}

Según los tipos de fotosíntesis, las plantas pueden dividirse en 3 tipos: C3, C4 y CAM. Las plantas del tipo CAM fueron excluidas del análisis, ya que las de CAM representan cactáceas. Los ciclos de Calvin-Benson o fase de fijación del $\mathrm{CO} 2$ de la fotosíntesis de las plantas $\mathrm{C} 3$ y C4 son diferentes. Entre las plantas $\mathrm{C} 3$, se obtiene la ecuación ajustada de la reacción: Ribulosa -1,5- bisfosfato + $\mathrm{CO} 2 \rightarrow 3$ - ácido fosfoglicérico para fijar el CO2. La reacción de las plantas $\mathrm{C} 4$ es: Ácido fosfoenolpirúvico+ $\mathrm{CO} 2 \rightarrow$ ácido oxaloacético.

Sin embargo en la atmósfera hay dos tipos de carbón, como $12 \mathrm{CO} 2$ y $13 \mathrm{CO} 2$. El porcentaje de $12 \mathrm{CO} 2$ es $98.89 \%$ y $13 \mathrm{CO} 21.11 \%$. Y $13 \mathrm{CO} 2 / 12 \mathrm{CO} 2=0.011225$ (Fig. 3). La planta fija $\mathrm{CO} 2$, tomando más $12 \mathrm{CO} 2$ de peso menor que $13 \mathrm{CO} 2$ de peso mayor. Y la proporción de $13 \mathrm{CO} 2 / 12 \mathrm{CO} 2$ se reduce. También las proporciones de las fases de fijación del $\mathrm{CO} 2$ de las plantas $\mathrm{C} 3$ y $\mathrm{C} 4$ son diferentes (Fig. 4). La proporción de fijación del $13 \mathrm{CO} 2 / 12 \mathrm{CO} 2$ de $\mathrm{C} 3$ es 0.010933 , mientras la de C4 0.011102. La planta de C4 tiene más 13C que la de C3. Los orgánicos de suelo provienen de plantas muertas que se descompusieron y condensaron poliméricamente. Y la proporción de 13CO2/12CO2 está afectada por la de las plantas originales.

\begin{tabular}{lcccc}
\hline & ${ }^{12} \mathrm{C}(\%)$ & ${ }^{13} \mathrm{C}(\%)$ & ${ }^{13} \mathrm{C} /{ }^{12} \mathrm{C}(R)$ & ${ }^{13} \mathrm{C}(\%)$ \\
\hline Atmósfera & 98.89 & 1.11 & 0.011225 & -1.07 \\
Planta C3 & 98.92 & 1.08 & 0.010933 & -27.07 \\
Planta C4 & 98.90 & 1.10 & 0.011102 & -12.03 \\
$\begin{array}{l}\text { PeeDee Belemnite } \\
\text { (Fósil director) }\end{array}$ & 98.889 & 1.111 & $0.011237\left(R_{\text {ref }}\right)$ & 0 \\
\hline
\end{tabular}

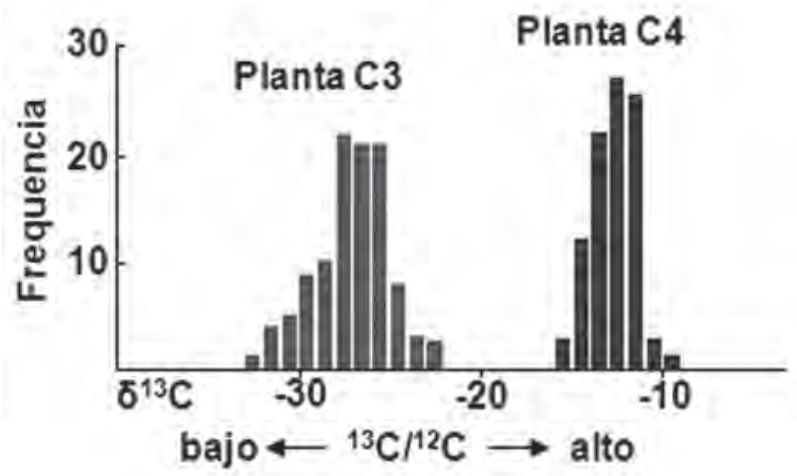

Figura 3. Abundancia natural de $\delta 13 \mathrm{C} \%$

a: Abundancia natural de $13 \mathrm{C}$ por $\delta 13 \mathrm{C} \%$ (Diels et al, 2001)

b: Abundancia natural de $813 \mathrm{C} \%$ de las plantas de $\mathrm{C} 3$ y $\mathrm{C} 4$ 


\begin{tabular}{|c|c|}
\hline Nelsunia canescens (1_am) Spreng. & -25.8 \\
\hline Physalis angulata Linn. & -264 \\
\hline Talimen triangulare (Jacq. I Willd. & -26.7 \\
\hline Corcherus chitorius Lim. & -26.8 \\
\hline Plovsalis micrantha Lim. & -27.0 \\
\hline Oldenlandia corimbesa Lim. & -27.4 \\
\hline Lindemia sp. Allioni & $-27,9$ \\
\hline Aculypha fimliriatu Schum. \& Thonn. & -27.9 \\
\hline Commelina benghalensis $\mathrm{L}$. & -28.0 \\
\hline Celosia leptoscaachya Benth. & $-2 \times .2$ \\
\hline Chromelacna celeruta (L., R.M. King \& Robimson & -28.2 \\
\hline Camme lina diffusa Burm 1, subsp, diffusa I.K. Mornon & $-2 x .3$ \\
\hline Laported aestrans (Linn, ) Chew. & $-2 \times 3$ \\
\hline Eupluerbia hetenophylla Linn. & -28.4 \\
\hline Vemonia cinerra (1.jun.) Lesh & -28.4 \\
\hline Tridax procumbens Linn. & -28.5 \\
\hline Spermucose ocymoides Burm. I. & -28.5 \\
\hline Antiaris africume Engl. & $-2 \times .6$ \\
\hline Syncirella nowiflem Gisenn. & $=2 \times 6$ \\
\hline Agernutum conysotides L.inn. & $-2 x .7$ \\
\hline Desmodium tortuosum (Sw, IX. & $-2 \times 7$ \\
\hline Solenessemon monostachyus (P. Beauy, Brig subsp, monnstachyus & $s-28.8$ \\
\hline Acanihusperverum haspidum $\mathrm{DC}$. & -28.9 \\
\hline Desomesleum scorpiurus (Sw) Desy. & $=29.1$ \\
\hline Glonixsa superisi Linn. & $=29.1$ \\
\hline Phsllanthes amaras Schisn. \& Thom. & -29.2 \\
\hline Paxsiflora foctias Linn. & -29.2 \\
\hline Cleome nitidosperma DC. & -29.4 \\
\hline 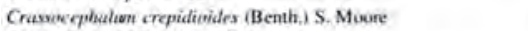 & -29.7 \\
\hline Desmestien triflenem Linn. DC. & 29.9 \\
\hline Mean $C_{3}$ species & $=2 \times .3$ \\
\hline
\end{tabular}

\begin{tabular}{|c|c|}
\hline Species & $\begin{array}{l}8^{\prime} \mathrm{C} \\
(x)+\end{array}$ \\
\hline Cypenes rotiondus Linn. & -10.0 \\
\hline Nytlinga bullosa Beauv, & 10.4 \\
\hline Paspaliam scrotriculasum linn & 10.7 \\
\hline Setaria barkata (Lam.) Kunih & -11.0 \\
\hline Dixitania horizontalis Willd. & 11.2 \\
\hline Engmsta riliaris (L.inn., R. Br. & 11.3 \\
\hline Marise as altemifolius Vahl & -12.4 \\
\hline Cynodem dactylom (Linn.) Perx & -12.4 \\
\hline Elensine indiva Gaerts. & 12.5 \\
\hline Dactyloen nuam argyptiam (Linn) P Beauv & $-12 . x$ \\
\hline Panicum maximum Jacq. & 12,9 \\
\hline Pertwlawo oleraces L.inn. & 13.5 \\
\hline Amanesihas verifis Linn. & 13.5 \\
\hline Boerhavia crecta Linn. & 13.5 \\
\hline Euphowbia hina Linn. & -13.7 \\
\hline Cuteris pilesa Schamach. & -14.3 \\
\hline Mean $C_{4}$ species & -12.2 \\
\hline
\end{tabular}

Ibadan, SW Nigeria $\left(7^{\circ} 30^{\prime} \mathrm{N}, 3^{\circ} 54^{\prime} \mathrm{E}\right)$

Figura 4. Valores de $\delta 13 \mathrm{C} \%$ de $\mathrm{C} 3$ y C4 en plantas (Diels et al. 2001)

En este estudio se mide la proporción de $13 \mathrm{CO} 2 / 12 \mathrm{CO} 2$ en el suelo del surco prehispánico para identificar el tipo de planta que cultivaba en la época prehispánica. La proporción de $13 \mathrm{C} / 12 \mathrm{C}$ en la muestra es Rsam. Y Rref es la proporción de $13 \mathrm{C} / 12 \mathrm{C}$ en fósil director de Pee Dee Belemnite. El valor de la proporción es $\delta 13 \mathrm{C}(\%)=($ Rsam $/$ Rref -1$) \times 1000$.

En la Figura 5 se muestran los valores de varias plantas en Ibadan, Nigeria. El valor de la planta C3 se ubica entre -25.8 y $-29.9 \%$, mientras el de C4 entre -10.0 y $-14.3 \%$. Figura 6 presenta un estudio de Australia. Se muestra la diferencia de valor de $\delta 13 \mathrm{C} \mathrm{( \% )} \mathrm{entre} \mathrm{varios} \mathrm{terrenos,} \mathrm{como} \mathrm{sabana,} \mathrm{selva}$ monzónica y pradera. Figura 6 se presenta un estudio de Francia. Se sacaron varias muestras de suelo con dos profundidades de $0-0.30 \mathrm{~m}$ y de $0.30-0.40 \mathrm{~m}$ en la milpa de maíz, la cual se deforestó y preparó para el cultivo. Las muestras de suelo se cernieron con tamices de varios tamaños antes del análisis. Para comparar los valores del suelo de milpa, se midieron las muestras de suelo de terreno forestal en sus cercanías. En la milpa de maíz el valor de $\delta 13 \mathrm{C}$ se aumentó distinguidamente entre -26 y - $12 \%$ más que en el terreno forestal. Así el análisis de $\delta 13 \mathrm{C}$ de suelo es muy efectivo para identificar el cultivo prehispánico entre las plantas de $\mathrm{C} 3$ y C4. 

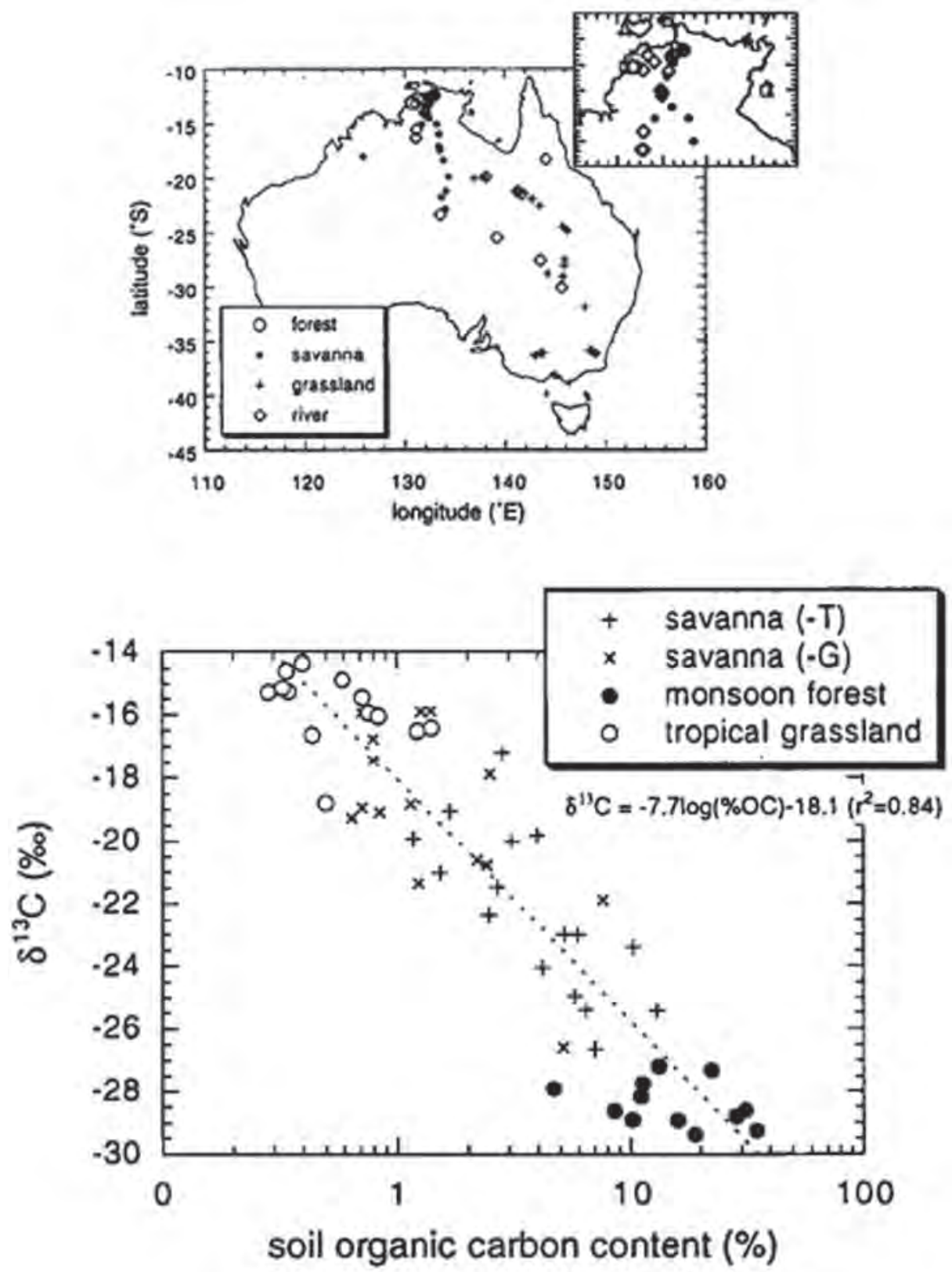

Figura 5. Carbón orgánico de suelo superficial (S.O.C.) y valores de $\delta$ 13C (Bird y Pousi 1997) 

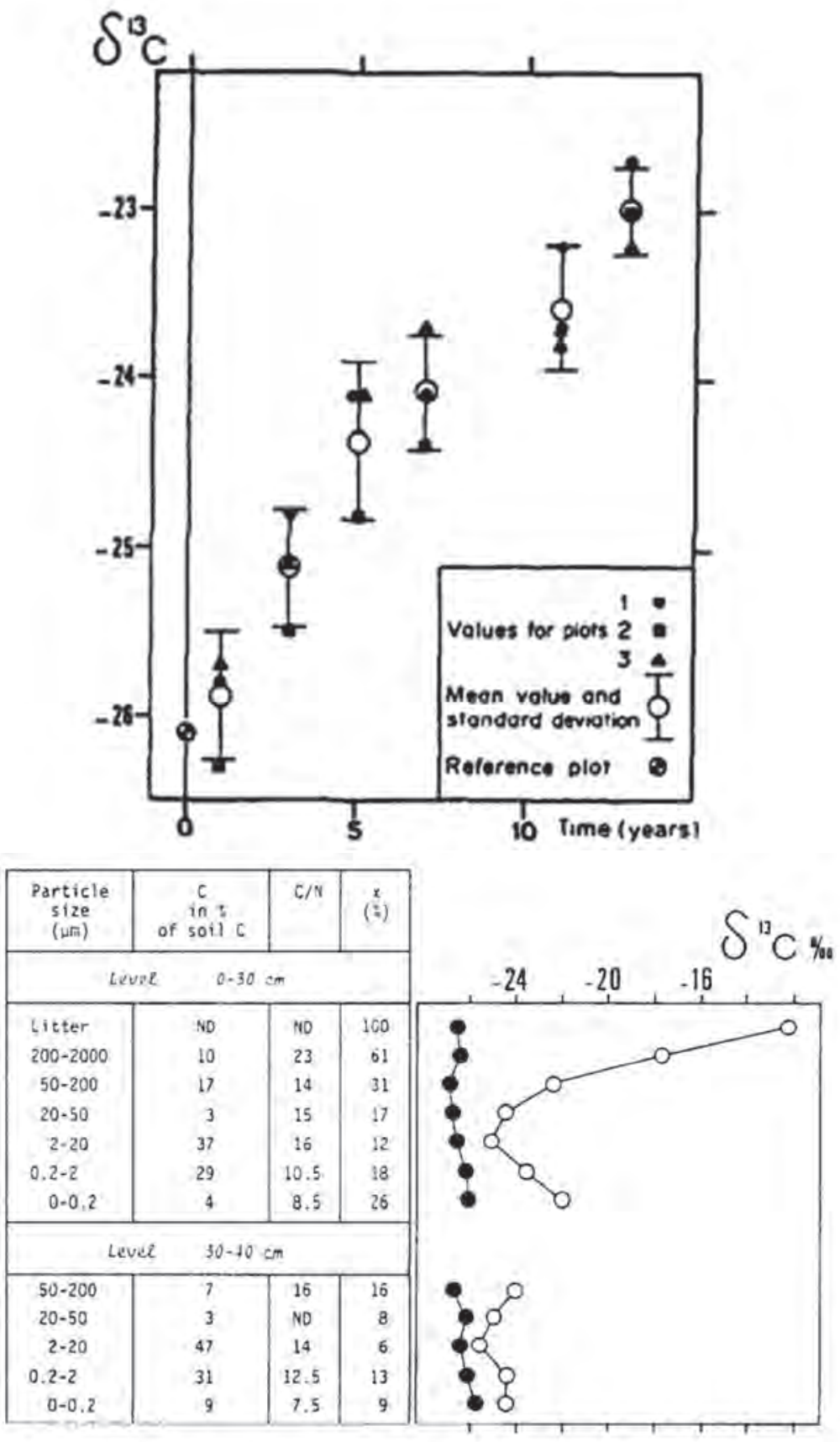

$x=$ percent of organic $C$ derived jrom corn

- jonzst soir

O cout cultivased soil (T)!

Figura 6. Maíz (Planta C4) en suroeste de Francia (Balesdent y Mariotti 1987) 


\section{Boulevard Monseñor Romero}

En el corte de que se ha hecho por la construcción de la carretera en Boulevard Monseñor Romero se encontraron unos surcos prehispánicos abajo de la capa de ceniza volcánica de T.B.J. Los surcos tienen forma ondulada. Entre los surcos miden un ancho de $0.89 \mathrm{~m}$, el cual se encuentra en la milpa actual. Abajo de la capa gruesa de ceniza volcánica, se encuentra una capa correspondiente al surco con una superficie de los surcos que consiste de la tierra amarillenta o grisácea, lo cual indica que sedimentaron por un tiempo suficiente y largo de cultivación. Sobre los surcos, se depositó flujo piroclástico de un grosor de más de $3 \mathrm{~m}$. Gracias a esta capa volcánica, se mantenían los característicos de la tierra de surco hasta la construcción moderna.

En la capa de los surcos se sacaron muestras de suelo de surco y de una profundidad de $0.40 \mathrm{~m}$ más abajo del surco, para el análisis a realizar entre los años de 2007 y 2009. Por el analizador de masa de isótopos estables (DELTA Plus-NC2500, Thermo Finnegan, San José, CA, USA), se midieron los valores de $\delta 13 \mathrm{C} \mathrm{( \% )} \mathrm{de} \mathrm{cada} \mathrm{muestra} \mathrm{de} \mathrm{suelo} \mathrm{(Fig.} \mathrm{7).}$

\begin{tabular}{|c|c|c|c|c|}
\hline \multicolumn{5}{|c|}{ ElSalvador Soiks } \\
\hline Sample & Ave C (\%) & Ave N(\%) & Ave CN & Ave $13 C$ \\
\hline 1 & 1.54 & 0.09 & 1810 & $-14,42$ \\
\hline 2 & 1.68 & 0.09 & 19.10 & -13.75 \\
\hline 3 & 209 & 0.10 & 21.83 & -1431 \\
\hline 4 & 197 & 0.09 & 21,41 & -14.10 \\
\hline 5 & 1.67 & 0.08 & 19.68 & -1457 \\
\hline 6 & 241 & 0.10 & 2337 & $-14,48$ \\
\hline 7 & 1.66 & 0.08 & 19.82 & -14.97 \\
\hline 8 & 1.59 & 0.08 & 1953 & -1486 \\
\hline 9 & 130 & 0.08 & 1537 & -1523 \\
\hline 10 & 1.80 & 0.10 & 1871 & $-14,66$ \\
\hline 11 & 245 & 0.11 & 21.92 & -1432 \\
\hline 12 & 152 & 0.08 & 1863 & $-14,44$ \\
\hline 13 & 1.16 & 0.07 & 1597 & -1455 \\
\hline 14 & 1.29 & 0.07 & 1825 & -1435 \\
\hline 15 & 0.62 & 0.06 & 1087 & -15.15 \\
\hline 16 & 0.68 & 0.06 & 10.95 & -1510 \\
\hline 17 & 086 & 0.06 & 13,40 & -13.61 \\
\hline \multicolumn{5}{|c|}{ SoilSamples in 2009} \\
\hline Sample & AveC (\%) & AveN(\%) & Ave.CN & Ave $13 \mathrm{C}$ \\
\hline 8 & 051 & 0.04 & 1324 & -1551 \\
\hline 9 & 0.18 & 0.02 & 9.07 & -16.60 \\
\hline 10 & 1.02 & 0.07 & 14.64 & $-19,04$ \\
\hline
\end{tabular}

Figura 7. Valores de $\delta 13 \mathrm{C} \%$ en Boulevard Monseñor Romero. 
La muestra del surco contiene $6.8-24.5 \mathrm{~g} / \mathrm{kg}$ de carbón, mientras la de $0.40 \mathrm{~m}$ de profundidad tiene solo $1.8-10.2 \mathrm{~g} / \mathrm{kg}$. Se deduce que se sedimentó una cantidad de materia orgánica por la agricultura. El valor de $\delta 13 \mathrm{C}(\%)$ de surco es entre -13.61 y $-15.23 \%$. El valor de $\delta 13 \mathrm{C}$ de $0.40 \mathrm{~m}$ de profundidad se encuentra entre -15.51 y $-19.04 \%$ más bajo que lo de surco, el cual se deduce que se cultivaba la planta de $\mathrm{C} 4$.

\section{Conclusión}

Se ha realizado el estudio de valor de $\delta 13 \mathrm{C}$ a través del analizador de masa de isótopos estables para identificar los cultivos de la época anterior a la erupción volcánica del Ilopango, ya que no se ha encontrado ningún material de flora o de cultivo. Por análisis de valor de $\delta 13 \mathrm{C}$, se puede decir que se cultivaba plantas del tipo C4 en la milpa de Boulevard Monseñor Romero, aunque no fue posible identificar la especie de la planta C4 por falta de información.

Dependiendo de la planta, se muestra el valor de $\delta 13 \mathrm{C}$ de suelo. Por este valor mismo se reconoce el tipo de la planta que se cultivaba o utilizaba para fertilizante. Finalmente será necesario reconocer la agricultura actual y prehispánica para estar al tanto que tipo de restos de plantas se quedan en la milpa después de la cosecha para confirmar el valor de $\delta 13 \mathrm{C}$ de tierra de los surcos.

\section{Referentes bibliográficos}

Amaroli, P. \& Robert, D. (1999). Milpas prehispánicas en El Salvador. En J. P. Laporte... et al. (Ed), XII Simposio de Investigaciones Arqueológicas en Guatemala, 1998. (562-572).

Balesdent, J., \& Mariotti A. (1987). Natural 13C Abundance as a Tracer for Studies of Soil Organic Matter Dynamics. Soil Biology and Biochemistry. 19(1), 25-30.

Bird, M. I., \& Pousai P. (1997). Variations of 613C in the surface soil organic carbon pool. Global Biogeochemical Cycles. 11(3), 313-322.

Diels, J., Vanlauwe, B., Sanginga, N., Coolen, E., \& Merckx, R. (2001). Temporal variations in plant $13 \mathrm{C}$ values and implications for using the 13C technique in long-term soil organic matter studies. Soil Biology and Biochemistry. 33(9), 1245-1251.

Earnest, H. H. (1976). Investigaciones efectuadas por el proyecto no. 1, programa de rescate arqueológico Cerrón Grande, en la hacienda Santa Bárbara, depto. de Chalatenango. Anales del Museo Nacional "David J. Guzmán. (49), 57-67.

Plunket, P., \& Uruñuela, G. (1998). Preclassic Household Patterns Preserved under Volcanic Ash at Tetimpa, Puebla, México. Latin American Antiquity 9 (4), 287-309.

Sheets, P. (2002). Befare the Volcanic Erupted: TheAncient Cerén Village in Central America., Austin: University of Texas Press

Xelhuantzi López, S. (2010). Seis muestras de sedimentos. En N. Íto (Ed), Casa Blanca, Chalchuapa, El Salvador. (233-236). 